\title{
Adaptación de la Escala Multidimensional de Orientaciones hacia la Deportividad al contexto del fútbol alevín
}

\author{
Adaptation of the Multidimensional Sportspersonship \\ Orientation Scale in Juvenile Football
}

\section{Adaptação da Escala Multidimensional de Orientaçóes face ao Desportivismo no contexto do futebol de formação}

\author{
J. Lamoneda Prieto ${ }^{1}$, F.J. Huertas Delgado ${ }^{2}$, L.G. Córdoba Caro ${ }^{1}$ y A.V. García Preciado ${ }^{1}$ \\ 1 Universidad de Extremadura, 2 E.U. de Magisterio "La Inmaculada"
}

\begin{abstract}
Resumen: El objetivo de este estudio es contextualizar la Escala Multidimensional de Orientaciones hacia la Deportividad al fútbol alevín. Participaron 160 jugadores de entre 10-12 años de edad. El instrumento mostró inicialmente una alta consistencia interna $(\alpha=0,77)$ pero no en la subdivisión en las cincosub-escalas sugeridas en la versión original $(\alpha<0,6)$. Esta circunstancia nos llevó a realizar una reducción dimensional en dos factores: personales y sociales. Para ello se realizó un análisis factorial y se contrastó con la bibliografía especializada. La versión definitiva consta de 21 ítems, subdivididos en las dos sub-escalas: goza de buena fiabilidad $(\alpha>0,6)$ y aceptable validez de constructo (KMO=0,657; $\chi^{2}=688,5 ;$ g.1. $\left.210 ; p<, 000\right)$. Palabras clave: evaluación, deportividad, fútbol base, propiedades psicométricas.

Summary: The objective of this study is to contextualize the Multidimensional Sportspersonship Orientation Scale in juvenile football. This research included 160 football players from 10 to 12 years of age. The instrument initially indicated high internal consistency $(\alpha=0.77)$, but did not show such high levels in the subdivision of the five sub-scales suggested by the original version $(\alpha<0.6)$. This finding led us to perform a dimensional reduction to two factors: personal and social. For that reason, a factor
\end{abstract}

analysis was performed and supported with relevant specialized literature. The final version consists of 21 items divided into two sub-scales: statistical reliability $(\alpha>0.6)$ and acceptable construct validity $\left(\mathrm{KMO}=0.657, \chi^{2}=\right.$ 688.5, df $210, \mathrm{p}<.000)$.

Keywords: Evaluation, Sportsmanship, Grassroots football, Psychometric properties.

Resumo: O objectivo deste estudo foi contextualizar a Escala Multidimensional de Orientaçôes face ao Desportivismo no futebol de formação. Participaram 160 jogadores entre os 10-12 anos de idade. O instrumento revelou inicialmente uma elevada consistência interna $(\alpha=0,77)$, contudo na subdivisão das cinco sub-escalas na versão original $(\alpha<0,6)$. Esta circunstância levou-nos a realizar uma redução dimensional em dois factores: pessoais e sociais. Para tal realizou-se uma análise factorial e comparou-se com a bibliografia especializada. A versão definitiva consta de 21 itens, subdivididos em duas sub-escalas: possui boa fidelidade $(\alpha<0,6)$ e aceitável validade de construto (KMO $=0,657 ; \chi 2=688,5$; g.l. $210 ; \mathrm{p}<, 000$ ).

Palavras-chave: avaliação, desportivismo, futebol de formação, propriedades psicométricas.

\section{Introducción}

El fútbol juega una un papel importante en la vida de un número elevado de niños y jóvenes. Es una pieza clave para el desarrollo físico, intelectual, emocional y social. Sin embargo si se aleja de una orientación educativa degenera en un deporte comercializado, selectivo, opresivo, lesivo, permisivo, agresivo y con falta de credibilidad. Por esta razón, numerosos trabajos resaltan la necesidad de plantear la iniciación deportiva de modo tal que garantice la correcta orientación del jugador en el proceso de formación, acercándose a lo que

\footnotetext{
Dirección para correspondencia [Correspodence address]: Javier Lamoneda Prieto. Doctorando en posesión del DEA Departamento de Didáctica de la Expresión Musical, Plástica y Corporal, Facultad de Educación, Universidad de Extremadura. Funcionario y profesor del IES Zaframagón. Avda. Manuel de Falla, s/n. 11690. Olvera (Cádiz) 655517918. E-mail: educacionfisicajlp@gmail.com
}

se ha denominado: “deporte educativo” (Mármol y Valenzuela, 2013).

La educación moral del futbolista ha de basarse en la transmisión de valores que sugiere el término "deportividad". Éste integra elementos tanto de ética individual como social. En el plano personal: el pleno compromiso por la práctica deportiva y a la vez, el control emocional para afrontar el error (Vallerand, Brière, Blanchard y Provencher, 1997); y social: el respeto a la normativa, a los adversarios y al árbitro (Comité Olímpico Internacional, 2001), la aplicación de principios de justicia para todos (Comité Internacional de Fair Play, 2012), la cortesía con el resto de participantes (Lemyre, Roberts y Ommudsen, 2002) y la ayuda al jugador lesionado, al que llega tarde a un partido o no dispone de material para practicar (Vallerand et al, 1997). 
En los últimos años han proliferado programas centrados en indagar cómo el ámbito de la actividad físico-deportiva puede contribuir al desarrollo socio-moral (Jacobs, Knoppers y Webb, 2013). Resulta interesante señalar que tales programas han de ser planificados y evaluados con el fin de darles credibilidad y convertirse en un modelo para otros. Para la valoración de los logros se han de emplear herramientas válidas, fiables y objetivas; económicas tanto en el gasto como en recursos humanos necesarios, con un procedimiento de ejecución sencillo y claro y que a su vez mida todas las partes e implicados en la intervención (Jiménez y Durán, 2004).

En el análisis de valores diferenciamos principalmente cinco métodos: foros científicos de opinión, entrevistas, cuestionarios-escalas y análisis de documentos y observación (Sáenz, 2010). De todos ellos, en el presente estudio nos centramos en las "escalas multidimensionales" por dar información de varias variables psicológicas de forma simultánea con el consiguiente beneficio en relación tiempo y esfuerzo (López-López, Jaenes-Sánchez y Cárdenas-Vélez, 2013). En particular nos detendremos en el "Multidimensional Sportspersonship Orientations Scale" (MSOS) diseñada por Vallerandet al. (1997) que validaron con una población canadiense de 362 deportistas (jugadores de bádminton, baloncesto, voleibol, natación y atletismo) y que consta de veinticinco ítems divididos en cinco dimensiones: (1) Compromiso con la práctica deportiva, (2) Convenciones sociales, (3) Respeto a las reglas, jueces y árbitros, (4) Respeto a los oponentes y (5) Perspectiva negativa. Los participantes deben manifestar su grado de acuerdo o desacuerdo en una escala tipo Likert con un rango de 1 a 5, siendo 1,"no se corresponde conmigo en absoluto"; 3, "se corresponde conmigo en parte" y 5 , "se corresponde exactamente conmigo".

El MSOS ha sido traducido a diferentes lenguas: noruega (Lemyreet al., 2002), española (Martín-Albo, Núñez, Navaro y González, 2006), griega (Pavlopoulou, Goniadou, Zachariadis y Tsormpatoudis, 2003), portuguesa (Serpa, Alves y Barreiros, 2004); utilizado en estudios de lengua francesa (Chantal, Soubranne y Brunel, 2009) y china (Shuge, 2011) y adaptado a disciplinas deportivas como en hockey (Dunn y Dunn, 1999).

La escala no ha estado exenta de críticas, principalmente por los problemas psicométricos que presenta (McCutcheon, 1999), especialmente en la quinta sub-escala que manifiesta unos índices bajos de consistencia interna. Shields y Bredemeier (1994) achacaron a la escala que un sesgo positivo debido a que cuenta con cuatro campos positivos y sólo uno negativo, además de no incluir aspectos tan relevantes como "ganar a toda costa". Finalmente, se ha cuestionado la presencia del compromiso en la escala, indicando que esa dimensión no tiene nada que ver con el juego limpio.

Para solventar las limitaciones planteadas, numerosos autores decidieron prescindir de la última sub-escala (Do- nahueet al., 2006; Fernandes, Vasconcelos-Raposo, Moreira y Costa, 2007; Gano-Overway, Guivernau, Magyar, Waldron y Ewing, 2005; Lemrey et al., 2002; Miller, Roberts y Ommundsen, 2004) y otros ampliar la escala a 30 ítems e incorporar una nueva dimensión, la "agresión instrumental" -comportamientos agresivos como el uso de la fuerza física- (Stornes y Bru, 2002). A la herramienta ampliada se denominó "versión extendida de la Escala Multidimensional de la deportividad” (EMSOS). Con los ajustes realizados demostraron mejores propiedades psicométricas que la herramienta original. La EMSOS fue utilizada por primera vez por Stornes y Bru (2002) y más tarde por Stornes y Ommundsen (2004). A pesar de los últimos ajustes realizados en la escala,también ha estado cuestionada su validez de constructo y fiabilidad (Knortz, 2009).

Aún a sabiendas de las limitaciones del MSOS (Knortz, 2009 y McCutcheon, 1999), la escala demuestra unos niveles adecuados de validez y fiabilidad. Ha sido utilizada en numerosas investigaciones: en el estudio del uso de sustancias prohibidas en el deporte (Barkoukis, Lazuras, Tsorbatzoudis y Rodafinos, 2011; Chantal et al., 2009; Donahue et al., 2006), para el análisis de la agresividad en el deporte (Chantal, Robin, Vernat y Bernache-Assollant, 2005), con el fin de examinar el papel de las variables predictoras como las metas de logro (Dunn y Dunn, 1999; Lemyre et al., 2002; Ryska, 2003); con el propósito de analizar la relación entre deportividad y sexo (Gutiérrez y Pilsa 2006), sexo y el nivel de competitividad (Proios et al., 2006) o sexo, edad y tipo de deporte (Zarotis, Psychountaki y Stavrou, 2009); para enfatizar la preocupación por respeto al árbitro (Joyner y Mummery, 2005; Kenworthy, 2010); para determinar la influencia de padres (Leo, Sánchez, Sánchez, Amado y García, 2009) o entrenadores (Kenworthy, 2010) en la deportividad de los futbolistas; en el estudio del papel del clima motivacional en la deportividad (Melchor y Ruiz, 2009; Miller et al.,2004; Ommundsen, Roberts, Lemyre y Treasure, 2003; Proios, 2010); en el análisis correlacional entre deportividad y motivación intrínseca (Nuñez, Martín-Albo, Navarro, Sánchez y González-Cutre, 2009), motivación hacia la deportividad (Pavlopoulou et al., 2003; Ryska, 2003; Vissoci, Viera y Lopes, 2008) o empatía (Sezen-Balçikanli y Yildiran, 2011).

Ninguna versión del MSOS contempla la realidad del fútbol base cuando puede ser un instrumento útil en el análisis de actitudes dentro del tratamiento formativo del jugador: al tratarse de una herramienta multidimensional de cómoda, rápida y económica aplicación, que posibilita obtener información de varias variables psicológicas de forma simultánea.

Dentro de las etapas formativas del jugador, la categoría alevín es un período representativo. Determina el último momento de adaptación del fútbol 7 a 11, el futbolista no participa aún en competiciones trascendentales y se encuentra a caballo entre la primera toma de contacto con el deporte 
y el salto al deporte especializado (Benítez, 2009). A nivel madurativo: ha desarrollado la personalidad y su capacidad de tratar información y de aprendizaje son excelentes (Ardá y Casal, 2003). Finalmente, desde el punto de vista moral, se encuentra en un nivel "convencional" (Kohlberg, 1992), que caracteriza tanto la etapa en la que se encuentra (pre-puberal), como la posterior (puberal).

Estas razones nos han llevado a buscar la especificidad en la evaluación de las orientaciones hacia la deportividad en la realidad del fútbol alevín. Se plantea como objetivo de este estudio validar la versión española de la escala MSOS (Martín-Albo et al., 2006) para muestras concretas de futbolistas de entre 10 y 12 años de edad, de forma que "tras la adaptación de la escala MSOS al fútbol alevín el instrumental goce de adecuadas propiedades psicométricas".

\section{Método}

\section{Participantes}

En el estudio 1 formaron parte 160 futbolistas con edades comprendidas entre 10 y 12 ańos $(\overline{\mathrm{x}} \overline{\mathrm{x}}=11, \mathrm{SD}=0,67)$. El género predominante fue el masculino $(98,72 \%)$. En el estudio 2 la participación se redujo a 126 jugadores $(\overline{\mathrm{x}} \overline{\mathrm{x}}=10,99, \mathrm{SD}=0,67)$ ya que 11 no asistieron a la segunda sesión de evaluación y 23 fueron desechados al no cumplimentar debidamente la escala: al entregar el documento incompleto, dar respuestas incongruentes - decantarse exclusivamente por puntuaciones extremas, valores 1 o 5, en todo el cuestionario-, o emplear una grafía que denotaba desinterés.

Se seleccionó una muestra que satisfaciese los siguientes requisitos: que fuesen futbolistas federados y que el tamaño de la misma permitiese aplicar técnicas estadísticas como las que requiere la validación de cualquier escala (análisis factorial). Todos los participantes procedían de clubes o asociaciones deportivas registradas en la Federación Gaditana de Fútbol y con sede en una ciudad de 200.000 habitantes al sur de España. De los veintiún clubes que cumplían estos requisitos se seleccionaron doce a través de la técnica de muestreo por conveniencia Para la selección de los jugadores se recurrió a la técnica de muestreo aleatorio simple por conglomerados estimando que el tamaño de la misma debía superar los 100 participantes para la aplicación del análisis factorial (Amesty, 2003). Las entidades procedían de categoría: preferente $(6,82 \%)$, primera provincial $(38,63 \%)$ y segunda provincial (54,54\%); a nivel demográfico, un $20 \%$ provenían de barrios en situación de exclusión social (Rodríguez, 2001) y un 5\% filiales de clubes inscritos en Liga de Fútbol Profesional.

En relación con las características socio-demográficas de los participantes se consideraron las siguientes variables: (1) La edad. (2) La condición socioeconómica de los familiares de los participantes: parados, obreros, pequeños empresarios, técnicos y profesionales. Se estableció como criterio registrar entre los dos progenitores la profesión de mayor rango en la escala. (3) El rendimiento del equipo a lo largo de la temporada, se registró en una escala de: muy bajo, "último, penúltimo o antepenúltimo"; bajo, " $4 \circ$ o $5^{\circ}$ o $6^{\circ}$ en orden inverso a la clasificación general"; medio, "todos los que no se incluyen en el resto de categorías"; alto " 4 o, $5^{\circ}$ o $6^{\circ}$ " y muy alto, "10, $2^{\circ}$ o 3 " (tabla 1).

Tabla 1. Características socio-demográficas de los participantes.

\begin{tabular}{lll}
\hline Muestra & & $\%$ \\
\hline \multirow{2}{*}{$\begin{array}{l}\text { Edad } \\
\text { (años) }\end{array}$} & 10 & $23,02 \%$ \\
& 12 & $55,56 \%$ \\
& Parados & $21,43 \%$ \\
\hline \multirow{3}{*}{ Condición socio-económica } & Obreros & $7,93 \%$ \\
& Pequeños Empresarios & $21,42 \%$ \\
(padre/madre) & Técnicos & $11,11 \%$ \\
& Profesionales & $11,90 \%$ \\
\hline \multirow{3}{*}{ Rendimiento } & Muy bajo & $25 \%$ \\
& Bajo & $8,33 \%$ \\
& Medio & $8,33 \%$ \\
& Alto & $25 \%$ \\
& Muy alto & $33 \%$ \\
\hline
\end{tabular}

\section{Instrumento}

La contextualización del instrumento a la realidad del fútbol base se fraguó en cuatro etapas sucesivas: (a) Adaptación del instrumento original al fútbol alevín, (b) Estudio 1: obtención inicial de datos y valoración de la consistencia interna de la escala y de las sub-categorías que la componen, (c) Reducción de la escala MSOS-F en dimensiones acordes con la lógica del futbolista alevín y estudio de la fiabilidad,(d) Estudio 2: obtención de una segunda muestra de datos y análisis de la fiabilidad.

a) Adaptación del instrumento original. La versión definitiva de la "Escala Multidimensional de Orientaciones hacia la deportividad adaptada al fútbol base (MSOS-F) consta de 21 ítems (Anexo 1). Para facilitar la comprensión del cuestionario a jóvenes futbolistas cada ítem de la versión española fue sustituido por una situación práctica de juego, por ejemplo: "no 2. Aplicar el máximo esfuerzo", por: "iTe esfuerzas al máximo en cada actividad, hasta en aquella que demanda un gran esfuerzo físico (opción 5)? o ¡eres de los que te quedas en la cola del grupo en el calentamiento (opción 1)?”.

Se substrajeron cuatro ítems de la versión española del MSOS, uno por no poderse aplicar en fútbol siete alevín: $n^{\circ}$ 
12 (Respetar las decisiones de otros jueces y árbitros) ya que en la categoría alevín solamente dirige el partido un árbitro; y tres por entender que existían una serie de ítems que guardaban gran similitud con otros ya incluidos en la escala: el $n^{\circ}$ 15 (respetar las reglas) similar al no 14 (Atenerse fielmente a todas las reglas del deporte); el no 10 (Ganando o perdiendo, darle la mano a un oponente) por estar incluido en el $n^{\circ} 6$ (Felicitar a un oponente tras una derrota) y no 9 (Felicitar a un oponente tras una victoria); y el no 25 (Enfurecerse por cometer un error) por interpretar que es similar al no 21 (No querer admitir los propios errores).

Finalmente, se redactó la última sub-escala de modo inverso a la versión original, de esta forma todos los ítems de la escala tienen unas puntuaciones bajas para atributos antideportivos y altos para orientaciones prodeportivas. Ésta circunstancia evita confusión en el encuestado y facilita el tratamiento de datos.

b) Estudio 1. Tras los ajustes realizados al modelo base, en una segunda fase se aplicó el instrumento a un grupo de 160 futbolistas. Los datos se trataron a través del paquete informático SPSS para Windows (versión 20.0) y se procedió al estudio de la fiabilidad de la escala y las cinco sub-escalas propuestas en la versión original. Para ello se recurrió al el análisis de la consistencia interna, utilizándose el coeficiente Alfa de Crombach que revela el grado en el que los ítems convergen. Han de alcanzar valores superiores a 0,6 para que posean fuerza buena (Garrido, Zagalaz y Romero, 2010).

c) Reducción dimensional y estudio de fiabilidad. Los resultados obtenidos en el estudio de la fiabilidad para las cinco sub-escalas no fueron satisfactorios, circunstancia que llevó a avanzar hacia una solución factorial inicial que aportase una nueva reducción dimensional, para lo que se recurrió la técnica de análisis factorial exploratorio. El método empleado fue el Análisis de Componentes Principales, que se considera un buen procedimiento para este tipo de estudios (Henson y Roberts, 2006; Widaman, 2007) y resultó ser el que generó la solución más razonable.

El esquema metodológico empleado puede sintetizarse en cuatro pasos: (1) Diagnóstico de condiciones previas necesarias. Para valorar la adecuación de la subdivisión de la escala se calcularon los estimadores de adecuación muestral de Kaiser-Meyer-Olkin y de significación estadística de Bartlett. (2) Extracción de factores. Se tomó la decisión de aplicar la reducción a dos dimensiones en aras de encontrar una dimensionalidad de factores previamente teorizados, en coherencia con la revisión bibliográfica: Aristóteles (trad. 1972), Cagigal (en Olivera, 2006), Carranza y Mora (2003), Martín (1987) o Aquino (trad. 1970). (3) Rotación de factores. Se aplicó el método de rotación ortogonal: normalización Varimax con Kaiser, ya que los factores correlacionaban de forma débil entre sí y el método al minimizar el número de variables que tienen saturaciones altas en cada factor, simplifica la interpre- tación de los datos. (4) Una vez obtenida la nueva reducción dimensional de la escala y de la interpretación de la misma, se procedió al estudio de la fiabilidad de la escala mediante el análisis de la consistencia interna.

d) Estudio 2. Tras un período de cinco meses, se volvió a pasar el MSOS-F a los mismos participantes pero en esta ocasión el número se redujo a 126 . De nuevo se procedió al análisis de la fiabilidad de las sub-escalas mediante el análisis del estadístico Alfa de Crombach.

\section{Procedimiento}

Previamente al estudio se solicitó consentimiento tanto a los representantes legales de los futbolistas cómo a las entidades deportivas. La recogida de datos se realizó inicialmente entre los meses de octubre y noviembre de 2011 con ocho clubes, y la final entre marzo y abril de 2012. Con el fin de mejorar el tamaño de la muestra se amplió el estudio a cuatro clubes más en la siguiente temporada 2012-13 en fechas similares a la anterior.

La administración de la MSOS-F se realizó bajo la presencia del investigador principal y siempre que hubo la posibilidad, en el vestuario del equipo. Los casos en los que no se contó con esta ubicación se habilitaron otras zonas como: bancos o vallas situadas en zonas colindantes al campo de entrenamiento.

Durante la administración de la escala se les comunicó verbalmente a los jugadores que debían dar su opinión personal sobre cada una de las situaciones planteadas con sinceridad y sin dejar ninguna cuestión sin responder. A cada jugador se le facilitó el material necesario para cumplimentar la escala, se les leyó cada ítem en voz alta y se les dejó tiempo para que respondiesen a cada cuestión. La principal duda que le generaba la herramienta fue graduar la respuesta en una escala (de 1 a 5) en lugar de en dos (sí o no). Se insistió que lo correcto era contestar de acuerdo con lo que ellos pensaban y que no siempre era necesario utilizar las respuestas extremas (1 ó 5). Los ítems que más preguntas generaron fueron los que formaban parte de la quinta sub-escala: antideportividad.

No hubo tiempo límite de respuesta, pero el tiempo aproximado en pasar la prueba fue de 15 minutos.

\section{Resultados}

\section{Fiabilidad}

La Escala MSOS-F obtuvo una fiabilidad (Alfa de Crombach) de 0,77. Al detenernos en el análisis de las cinco categorías en la que se subdividió originalmente la escala (Vallerand et al., 1997) observamos cómo los datos oscilaron entre 0,51 y 0,59 (tabla 2). 
Tabla 2. Valores de consistencia interna (en diagonal) y correlacciones de Pearson.

\begin{tabular}{lccccc}
\hline & 1 & 2 & 3 & 4 & 5 \\
\hline 1. Compromiso & $(, 59)$ &, 269 &, 474 &, 341 &, 222 \\
2. Convecciones sociales & & $(, 57)$ &, 403 &, 394 &, 104 \\
3. Respeto a la normativa & & & $(, 53)$ &, 364 &, 177 \\
4. Respeto al oponente & & & & $(, 51)$ &, 332 \\
5. Antideportividad & & & & & $(, 51)$ \\
\hline
\end{tabular}

Se presentan además, las correlaciones entre las cinco subescalas a través del coeficiente de Pearson para evaluar la validez de constructo de la escala. Los resultados indican que las correlaciones son positivas y moderadas. Los valores más bajos se hallaron siempre con la quinta sub-escala (antideportividad).

\section{Análisis factorial}

Indicadores de Kaiser-Meyer-Olkin $\mathrm{KMO}=0,657$; prueba de esfericidad de Barlett: $\chi^{2}=688,5$; g.1. 210 y $p=, 000$.

Se obtuvo una estructura dimensional de dos componentes y 21 ítems, que explicaron el 27,60\% de la varianza. Todos los ítems alcanzaron pesos factoriales en el factor de pertenencia entre 0,3 y 0,6, a excepción delos ítems: $\mathrm{n}^{\mathrm{o}} 1,10$ y 11 que sus cargas fueron significativas en la dimensión opuesta pero que fueron incluidas en las de menos adherencia por motivos de contenido.

Tabla 3. Media, desviación estándar, análisis factorial y alfa.

\begin{tabular}{lcccc}
\hline & M & SD & Factor 1 & Factor 2 \\
\hline 1. Asistencia & 4,12 & 1,00 &, 102 & \\
2. Esfuerzo & 4,03 & 1,06 &, 360 & \\
3. Al errar, pensar cómo mejorar & 4,11 & 1,00 &, 431 & \\
4. Al fallar un gol seguir concentrado & 3,98 & 1,16 &, 383 & \\
5. Jugar con ilusión... & 4,34 & 0,94 &, 353 & \\
12. Honestidad en el juego & 3,78 & 1,34 &, 521 & \\
18. Enfadarse o admitir los errores & 3,74 & 1,38 &, 331 & \\
19. Jugar por ganar o por diversión & 3,56 & 1,34 &, 408 & \\
20. Poner excusas cuando pierdes & 3,69 & 1,19 &, 329 & \\
21. Protestar al entrenador & 4,37 & 1,07 &, 310 & \\
6. Dar la mano al vencedor & 4,17 & 1,21 & &, 433 \\
7. Dar la mano al entrenador oponente & 2,39 & 1,50 & &, 521 \\
8. Felicitar al oponente & 2,76 & 1,60 & &, 573 \\
9. Animar al perdedor & 3,90 & 1,48 & &, 540 \\
10. Obedecer al árbitro & 3,98 & 1,20 & &, 274 \\
11. Respetar al árbitro & 3,09 & 1,29 & & -120 \\
13. Permitir jugar al que llega tarde & 3,98 & 1,33 & &, 461 \\
14. Preocuparse por el lesionado & 4,03 & 1,12 & &, 538 \\
15. Rectificar una situación injusta & 1,78 & 1,25 & &, 275 \\
16. Prestar material deportivo & 2,64 & 1,59 & &, 512 \\
17. Rechazar un gol y atender lesionado & 3,33 & 1,51 & &, 468 \\
Varianza & & & $15,25 \%$ & $27,60 \%$ \\
\hline
\end{tabular}

\section{Fiabilidad en pretest y post test}

La tabla 4 muestra valores de consistencia interna superiores a 0,6 en las dos mediciones realizadas. Los resultados fueron mejores en el segundo estudio que en el primero. La dimensión II (componentes sociales) puntuaciones superiores que la I (componentes personales).
Tabla 4. Valores alfa de la escala MSOS-F y de las sub-escalas I y II.

\begin{tabular}{lcc}
\hline & Estudio 1 & Estudio 2 \\
\hline Componentes personales &, 610 &, 694 \\
II. Componente sociales &, 701 &, 722 \\
MSOS-F &, 758 &, 827 \\
\hline
\end{tabular}




\section{Discusión y conclusiones}

Diversos autores argumentan la necesidad de la adecuación muestral de los instrumentos de evaluación (Gimeno, Buceta y Pérez-Llantada, 2007). "En la búsqueda de la especificidad suelen realizarse adaptaciones de instrumentos genéricos a muestras más concretas" (López-López et al., 2013; p. 22). En este caso se realizaron adaptaciones en la redacción de cada ítem y el tamaño de la escala. Los resultados mostraron un instrumento que consta de 21 ítems frente a los 25 iniciares, con una estructura final de dos sub-escalas a diferencia de las cinco del MSOS original.

El estudio de la consistencia interna del instrumento reveló que la escala goza de fiabilidad en el estudio de la deportividad pero que no era viable la subdivisión en las categorías sugeridas en anteriores investigaciones. Lemyre et al. (2002) encontraron una aceptable consistencia interna, con valores entre 0,68 y 0,76 para el alfa de Cronbach, excepto en la sub-escala de Enfoque Negativo, que no obtuvo un nivel adecuado de fiabilidad $(\alpha=0,39)$. Martín-Albo et al. (2006) realizaron una doble prueba con una muestra de 253 deportistas canarios, con una media de edad de 21 ańos, participantes en 17 deportes diferentes, obteniendo buenos resultados en todos los campos (oscilaban entre 0,71 y 0,86$)$ a excepción del quinto $(\alpha=0,60$ y 0,56$)$. Proios (2010) aplicó la versión griega (Pavlopoulou et al., 2003) con una población de 214 estudiantes de escuelas deportivas de entre 12 y 18 ańos y obtuvo unos resultados que oscilaban entre 0,66 y 0,76 a excepción de la perspectiva negativa $(\alpha=0,46)$. La versión portuguesa se le atribuye a Serpa et al. (2004), fue utilizado en el mismo idioma por Vissoci et al. (2008) y Andaki (2012). Los resultados del último estudio realizado en lengua portuguesa con una muestra de 215 participantes de una edad media de 16 años de nacionalidad brasileña, paraguaya y guatemalteca oscilaron entre 0,67 y 0,80 a excepción de la perspectiva negativa $(\alpha=0,51)$.

Las dos razones que presumiblemente pueden justificar los valores de consistencia interna obtenidos en este trabajo $(\alpha<$ $0,6)$ son: primera, que la escala original fue diseñada para el estudio de la deportividad en cualquier disciplina deportiva y en nuestro caso se adaptó al fútbol; y segunda, que los participantes en el programa fueron niños/as cuando el diseño de la escala original se aplicó al ámbito universitario (Vallerand et al., 1997) y en posteriores trabajos la edad media de los participantes nunca fue menor de los doce años. Estas razones nos sugirieron plantear un análisis factorial que nos permitiera realizar nuevas agrupaciones desde la lógica del futbolista de categoría alevín.

El resultado de la nueva agrupación sugiere diferenciar dos dimensiones: (1) Componentes de ética personal y (2) Componentes de ética social. Los componentes personales incluyen a las sub-escalas del modelo original no 1 y 5 (compromiso con la práctica deportiva y antideportividad).
Los componentes sociales integran a las sub-escalas $n^{\circ} 2$, 3 y 4 (convecciones sociales, respeto a la normativa jueces y árbitros y respeto a los oponentes) a excepción del ítem no 12 (honestidad en el juego) que aún perteneciendo a la sub-escala 3 se incluyó en el bloque relacionado con factores personales atendiendo a su carga factorial y criterios conceptuales.

La reducción de la escala a factores personales y sociales es coherente con la bibliografía revisada. Cagigal (en Olivera, 2006) diferenció dentro de los valores del deporte los relacionados con la ética individual (búsqueda de la perfección, menosprecio al peligro y dedicación íntegra) y aquellos vinculados con la ética social (colaboración, compañerismo, amistad, obediencia, autoridad y justicia). Carranza y Mora (2003), basándose en Puig (1993) y Gutiérrez Sanmartín (1995) plantearon el estudio de valores en el área de educación física diferenciando valores personales (voluntad de valor, capacidad de crítica y autocrítica, autonomía y responsabilidad) y sociales (imparcialidad, diversión, participación lúdica, mejora de la salud, aventura y riesgo, creatividad y autodisciplina). Martín (1987) por su parte, diferenció cuatro tipos de valores importantes en programas de educación moral: individuales (veracidad, honestidad, disciplina, tolerancia, etc.), sociales (cooperación, rectitud, afabilidad, justicia, respeto, etc.), del país y el mundo (patriotismo, conciencia nacional, civismo, etc.) y de proceso (enfoque científico de la realidad, discernimiento, búsqueda de la verdad, reflexión, etc.).

Desde la ética de las virtudes también se contempla la subdivisión en dos bloques: el primer bloque lo integran las virtudes morales que dependen del autodominio. Aristóteles (trad. 1972) las denomina "virtudes éticas o del carácter propias del autodominio" y Tomás de Aquino (trad. 1970) "virtudes morales que perfeccionan el apetito inferior". Pertenecen a este grupo: la templanza, la fortaleza y todas las virtudes anejas a la fortaleza, como son, según Tomás de Aquino: paciencia, perseverancia, magnanimidad y magnificencia. Y el segundo bloque, las virtudes morales propias de las relaciones humanas. Aristóteles las denomina "virtudes éticas o del carácter propias de las relaciones humanas" y Tomás de Aquino "virtudes morales del entendimiento práctico, que perfecciona el apetito superior o voluntad". La justicia es considerada por estos autores la principal de las virtudes morales, fundamento del orden social humano, que da armonía a las demás cualidades. Por ello, se toma como virtud principal de la que parten otras como: la generosidad/liberalidad, amabilidad, veracidad, buen humor, afabilidad/dulzura/mansedumbre y némesis.

Entre las limitaciones de la adaptación realizada de la escala de Martín-Albo et al. (2006) cabe destacar el reducido ámbito de aplicación a la categoría alevín. Por ello, sería idónea su implementación en el resto de etapas formativas del futbolista y analizar la valía del instrumental. No obstante, la simplicidad de la redacción de cada ítem sugiere que pueda 
ser de utilidad en el resto de categorías que conforman el fútbol base.

Otra salvedad es el necesario apoyo en la bibliografía específica para justificar la nueva reducción dimensional: al no haberse hallado pesos factoriales satisfactorios en todos los ítems que componen la escala, ni un porcentaje alto de explicación de la varianza para esta estructura dimensional.

En relación con los valores obtenidos en el estudio de la fiabilidad para las cinco sub-escalas que sugiere la versión original los autores argumentan que la subdivisión no se ajustaba a la realidad del fútbol base. Convendría indagar en esta línea con el fin de explicar detenidamente a qué se debe esta circunstancia.

La principal limitación que presenta este estudio es la dificultad inherente a la medición de los valores que presentan los deportistas. No se trata de un elemento que podamos analizar de forma directa, sino que lo debemos hacer a través de la declaración que se realiza mediante un cuestionario. De esta forma, se debe seguir trabajando en la elaboración y adaptación de instrumentos válidos que permitan obtener unos resultados lo más fiables posibles sobre los valores que presentan los deportistas.
Las características psicométricas de validez de constructo atendiendo a toda la muestra sin distinción de sub muestras son adecuadas, así como la subdivisión en dos escalas. Este resultado nos permite ofrecer un instrumento que aporta: rápida aplicación, al contar con tan solo 21 ítems; análisis sencillo, tan sólo se subdivide en dos dimensiones: factores personales y sociales; adaptado a jóvenes deportistas (menores de 12 ańos), al simplificarse la redacción de cada ítem; y dirigido específicamente al contexto del fútbol.

\section{Aplicaciones Prácticas}

Por tanto, el presente trabajo cobra especial importancia ya que dota a los entrenadores de un instrumento que permite analizar los valores que presentan sus jugadores mediante un cuestionario sencillo, con el fin de poder trabajar en la línea de una educación a través del deporte. Es importante incidir en este aspecto en la formación que se realiza de los entrenadores a través de las diferentes diputación provinciales.

\section{Anexo. Escala Multidimensional de Orientaciones hacia la Deportividad adaptada al fútbol base (MSOS-F)}

Expresa el grado de acuerdo o desacuerdo con cada una de las siguientes cuestiones con una puntuación desde 1 a 5 . Por favor, se sincero y no te olvides de contestar todas las preguntas.
(1) No se corresponde conmigo en absoluto

(2) Prácticamente no se corresponde conmigo

(3) Se corresponde conmigo en parte

(4) Se corresponde conmigo en gran medida

(5) Se corresponde exactamente conmigo

\begin{tabular}{|c|c|c|c|c|c|}
\hline \multicolumn{6}{|l|}{ I. Factores personales } \\
\hline 1. ¿̇Asistes rigurosamente a todos los entrenamientos y partidos (opción 5)? o ¿`faltas mucho (opción 1)? & 1 & 2 & 3 & 4 & 5 \\
\hline $\begin{array}{l}\text { 2. ¿Te esfuerzas al máximo en cada actividad, hasta en aquella que exige un gran esfuerzo físico (opción 5)? o ¿eres } \\
\text { de los que te quedas en la cola del grupo en el calentamiento (opción 1)? }\end{array}$ & 1 & 2 & 3 & 4 & 5 \\
\hline 3. Cuando cometes un error, ¿piensas como mejorar (opción 5)? o ¿simplemente te enfadas (opción 1)? & 1 & 2 & 3 & 4 & 5 \\
\hline $\begin{array}{l}\text { 4. Cuando fallas una clara ocasión de gol, ¿̨rápidamente te olvidas y estas atento a la siguiente jugada (opción 5)? o } \\
\text { ¿te lamentas (opción 1)? }\end{array}$ & 1 & 2 & 3 & 4 & 5 \\
\hline $\begin{array}{l}\text { 5. Cuando te enfrentas a un equipo muy bueno y sabes de antemano que vas a perder, ¿juegas con ilusión y entre- } \\
\text { ga (opción 5)? o ¿te desanimas antes de empezar (opción 1)? }\end{array}$ & 1 & 2 & 3 & 4 & 5 \\
\hline $\begin{array}{l}\text { 6. ¿Nunca utilizas las normas para beneficio propio (opción 5)? o ¿̨recurres a la trampa como fingir una caída para } \\
\text { que piten una falta e incluso un penalti por caer al borde del área rival (opción 1)? }\end{array}$ & 1 & 2 & 3 & 4 & 5 \\
\hline $\begin{array}{l}\text { 7. ¿Admites tus errores sin enfadarte (opción 5)? o ¿eres de los que te enfadas muchísimo cuando te corrigen } \\
\text { (opción 1)? }\end{array}$ & 1 & 2 & 3 & 4 & 5 \\
\hline $\begin{array}{l}\text { 8. ¿Juegas por diversión (opción 5)? o ¿compites por ganar y en un futuro obtener grandes recompensas económi- } \\
\text { cas (opción 1)? }\end{array}$ & 1 & 2 & 3 & 4 & 5 \\
\hline $\begin{array}{l}\text { 9. ¿Eres de los que de los que admiten la derrota (opción 5)? o ¿̨de los ponen excusas cuando pierden o juegan mal } \\
\text { (opción 1)? }\end{array}$ & 1 & 2 & 3 & 4 & 5 \\
\hline $\begin{array}{l}\text { 10. ¿Obedeces a tu entrenador sin protestar (opción 5)? o por el contrario ¿protestas sus correcciones o decisiones, } \\
\text { por ejemplo cuando te pide que juegues en una posición que no te gusta o realizas un ejercicio que no te apetece } \\
\text { (opción 1)? }\end{array}$ & 1 & 2 & 3 & 4 & 5 \\
\hline
\end{tabular}




\begin{tabular}{|c|c|c|c|c|c|}
\hline \multicolumn{6}{|l|}{ II. Factores sociales } \\
\hline $\begin{array}{l}\text { 11. Cuando pierdes claramente un partido, ¿le das la mano al vencedor admitiendo la derrota (opción 5)? o ¿̇te vas } \\
\text { rápidamente al vestuario enfadado sin ganas de hablar ni dar la mano a nadie (opción 1)? }\end{array}$ & 1 & 2 & 3 & 4 & 5 \\
\hline $\begin{array}{l}\text { 12. Al terminar el partido, ¿le das la mano al entrenador del equipo contrario (opción } 5 \text { )? o ¿̇te vas rápidamente al } \\
\text { vestuario sin despedirte (opción } 1 \text { )? }\end{array}$ & 1 & 2 & 3 & 4 & 5 \\
\hline $\begin{array}{l}\text { 13. Si durante el juego un futbolista del otro equipo hace una jugada fantástica, ¿le felicitas (opción 5)? o ¿te enfa- } \\
\text { das y no admites el buen juego del equipo contrario (opción 1)? }\end{array}$ & 1 & 2 & 3 & 4 & 5 \\
\hline $\begin{array}{l}\text { 14. Cuando ganas un partido, ¿̇animas a los jugadores del otro equipo que ha perdido?, no se trata solo de darle la } \\
\text { mano, sino por ejemplo decirle que han jugado muy bien a pesar de perder (opción 5). O por el contrario, ¿̇muestras } \\
\text { una actitud de indiferencia e incluso de desconsideración (opción 1)? }\end{array}$ & 1 & 2 & 3 & 4 & 5 \\
\hline $\begin{array}{l}\text { 15. Cuando el árbitro pide que te coloques más atrás en una barrera, que respetes la línea de banda en un saque o } \\
\text { que abandones el campo, ¿̨le obedeces sin protestar (opción 5)? o ¿̨criticas sus decisiones faltándole al respeto a través } \\
\text { de un mal gesto o una mala contestación (opción 1)? }\end{array}$ & 1 & 2 & 3 & 4 & 5 \\
\hline $\begin{array}{l}\text { 16. Cuando el árbitro se equivoca claramente en tu contra, ¿̨le respetas (opción 5)? o ¿̨te enfureces y protestas (op- } \\
\text { ción 1)? }\end{array}$ & 1 & 2 & 3 & 4 & 5 \\
\hline $\begin{array}{l}\text { 17. ¿Permitirías jugar a un futbolista del otro equipo que ha llegado tarde (opción 5)? o ¿̨le impedirías jugar porque } \\
\text { beneficia a tu equipo (opción 1)? }\end{array}$ & 1 & 2 & 3 & 4 & 5 \\
\hline $\begin{array}{l}\text { 18. Cuando un jugador del otro equipo se lesiona, ¿te acercas a él para preocuparte por su situación?, ¡tratas de } \\
\text { ayudarlo (opción 5)? o ¿te despreocupas y simplemente esperas a que el árbitro reanude el juego (opción 1)? }\end{array}$ & 1 & 2 & 3 & 4 & 5 \\
\hline $\begin{array}{l}\text { 19. Imagina que durante un partido le das al balón con la mano y el árbitro no se da cuenta, ¿qué harías?: ¿habla- } \\
\text { rías con él para que rectifique ya que es injusto para el otro equipo (opción 5)? o ipermanecerías callado porque } \\
\text { perjudica a tu equipo (opción 1)? }\end{array}$ & 1 & 2 & 3 & 4 & 5 \\
\hline $\begin{array}{l}\text { 20. Si estas en el banquillo sin jugar, ¿serías capaz de dejarle tus espinilleras o botas a un futbolista del otro equipo } \\
\text { que se le han olvidado para que pueda jugar (opción 5)? o ¿nunca le dejarías tu equipación (opción 1)? }\end{array}$ & 1 & 2 & 3 & 4 & 5 \\
\hline $\begin{array}{l}\text { 21. ¿Echarías el balón fuera para que atendiesen a un jugador lesionado, renunciando a una clara ocasión de gol } \\
\text { (opción 5)? o ¿̇marcarías el gol (opción 1)? }\end{array}$ & 1 & 2 & 3 & 4 & 5 \\
\hline
\end{tabular}

\section{Bibliografía}

1. Amesty, S.C. (2003). Barriers to physical activity in the Hispanic Community. Journal of Public Health Policy, 24(1), 41-58.

2. Andaki, R. (2012). Fair play: instrumentos para avaliação e as orientaçóes desses valores no comportamento de jovens atletas. Programa de Pós-Graduação em Educação Física, para obtenção do título de Magister Scientiae. Universidade Federal de Viçosa, Minas Gerais (Brasil).

3. Aquino, T. (1970). Suma Teológica. Madrid: Editorial Católica.

4. Ardá, T. y Casal, C. (2003). Metodología de la enseñanza del fútbol. Paidotribo: Barcelona.

5. Aristóteles (1972). Moral, a Nicómaco. Madrid: Espasa-Calpe (5Ed.).

6. Barkoukis, V., Lazuras, L., Tsorbatzoudis, H., y Rodafinos, A. (2011). Motivational and sportspersonship profiles of elite athletes in relation to doping behavior. Psychology of Sport \& Exercise, 12(3), 205-212.

7. Benítez, S. (2009). Metodología y niveles de enseñanza en la iniciación deportiva al fútbol. Revista Digital efdeportes, 13(129). Recuperado de: http://www.efdeportes.com/efd129/metodologia-de-ensenanza-en-lainiciacion-deportiva-al-futbol.htm

8. Carranza, M., y Mora, J. M. (2003). Educación física y valores: educando en un mundo complejo: 31 propuestas para los centros escolares. Barcelona: Grao.

9. Chantal, Y., Robin, P., Vernat, J.P., y Bernache-Assollant, I. (2005) Motivation, sportspersonship, and athletic aggression: a mediational analysis. Psychology of Sport and Exercise, 6, 215-232.

10. Chantal, Y., Soubranne, R., y Brunel, P. (2009). Exploring the social image of anabolic steroids users through motivation, sportspersonship orientations and aggression. Scandinavian Journal Of Medicine \& Science In Sports, 19(2), 228-234.
11. Comité Internacional de Fair play (2012). The essence of fair play and history. En Web: http: //www.fairplayinternational.org.

12. Comité Olímpico Internacional (2001). Manual de Administración Deportiva. Solidaridad Olimpica. Lausana: Comité Olímpico Internacional.

13. Donahue, E.G., Miquelon, P., Valois, P., Goulet, C., Buist, A., y Vallerand, R. J. (2006). A motivational model of performance-enhancing substance use in elite athletes. Journal of Sport and Exercise Psychology, 28(4), 511-520.

14. Dunn, J.G.H., y Dunn, J.C. (1999). Goal Orientations, Perceptions of Aggression, and Sportspersonship in Elite Male Youth Ice Hockey Players. The Sport Psychologist, 13, 183-200.

15. Fernandes, H., Vasconcelos-Raposo, J., Moreira, M., y Costa H.A. (2007). Influência das orientaçôes motivacionais nas atitudes desportivas em aulas de Educação Física. Revista Motricidade, 3(3), 16-23.

16. Gano-Overway, L.A., Guivernau, M., Magyar, T.M., Waldron, J.J., y Ewing M.E. (2005). Achievement goal perspectives, perceptions of the motivational climate and sportspersonship: individual and team effects. Psychology of Sport \& Exercise, 6, 215-232.

17. Garrido, M. E., Zagalaz, M. L., y Romero, S. (2010). Diseńo y validación de un cuestionario para técnicos deportivos acerca de su opinión sobre las actitudes de padres y madres en el deporte (CTPMD). Cuadernos de Psicología del Deporte, 10(2), 7-21.

18. Gimeno, F., Buceta, J. M. y Pérez-Llantada, M.C. (2007). Influencia de las variables psicológicas en el deporte de competición: evaluación mediante el cuestionario Características psicológicas relacionadas con el rendimiento deportivo. Psicothema, 19(4), 666-671. 
19. Gutiérrez, M., y Pilsa, C. (2006). Orientaciones hacia la deportividad de los alumnos de educación física. Apunts: PUNTS Educación Física y Deportes, $864^{\circ}$ trimestre, 86-92.

20. Henson, R.K. y Roberts, J.K. (2006). Use of exploratory factor analysis in published research: common errors and some comments on improved practice. Educational and Psychological Measurement, 66, 393-416.

21. Jacobs, F., Knoppers, A., y Webb, L. (2013).Making sense of teaching social and moral skills in physical education. Physical Education y Sport Pedagogy, 18, 1-14. DOI: 10.1080/17408989.2011.621118.

22. Jiménez, P.J. y Durán, L.J. (2004).Propuesta de un Programa para Educar en Valores a través de la Actividad Física y el Deporte. Apunts: Educación Física y Deportes, 77, 25-29.

23. Joyner, K., y Mummery, K. (2005).Influences on sportspersonship orientations: Contextual and motivational. Youth Studies Australia, 24, 48-53.

24. Kenworthy, L.B. (2010). Actual and Perceived Coaches' Sportspersonship Behaviours and their Relationship with Young Athletes' Sportspersonship Orientations. (Thesis).Faculty of Applied Health Sciences, Brock University, St. Catharines, Ontario.

25. Knortz, G.S. (2009). A case study: assessing the validity and reliability of the multidimensional sportspersonship orientation scale among college athletes.(Thesis) The Faculty of the Graduate College, University of Vermont, Vermont.

26. Kohlberg, L. (1992): Psicología del desarrollo moral, Bilbao, Desclée de Brouwer, 1984.

27. Lemyre, P.N., Roberts, G.C., y Ommundsen, Y. (2002). Achievement goal orientations, perceived ability, and sportspersonship in youth soccer. Journal Applied Sport Psychology, 14, 120-136.

28. Leo, F.M., Sánchez, P. A., Sánchez, D., Amado, D. y García, T. (2009). Interacción entre la percepción del comportamiento de los padres y los comportamientos deportivos en edades de iniciación. Acción psicológica, 6(2), 55-62.

29. López-López, I.S., Jaenes-Sánchez, J.C., y Cárdenas-Vélez, D. (2013). Adaptación para futbolistas (CPRD-F) del cuestionario "características psicológicas relacionadas con el rendimiento deportivo". Cuadernos de Psicología del Deporte, 13(2), 21-30.

30. Mármol, A.G., y Valenzuela, A.V. (2013). Análisis de la idea de deporte educativo. Revista de Ciencias del Deporte, 9(1), 47-57.

31. Martín (1987), citado en Gutiérrez Sanmartín (2003). Manual sobre valores en la educación física y el deporte. Barcelona: Paidós.

32. Martín-Albo, L. J., Núńez Alonso, J. L., Navarro Izquierdo, J. G., y González Ruíz, V. M. (2006). Validación de la versión española de la Escala Multidimensional de Orientaciones a la Deportividad. Revista de Psicología del Deporte, 15(1), 9-22.

33. McCutcheon, L.E. (1999). The multidimensional sportspersonship orientations scale has psychometric problems. Journal of Social Behavior and Personality, 14, 439-444.

34. Melchor G. y Ruiz, L. M. (2009). Perceived motivational climate, sportsmanship, and students' attitudes toward physical education classes and teachers. Perceptual and Motor Skills, 108, 308-326.

35. Miller, B.W., Roberts, G.C., y Ommundsen, Y. (2004).Effect of motivational climate on sportspersonship among competitive male and female football players. Scandinavian Journal of Medicine and Science in Sports, Copenhagen, 14, 193-202.

36. Mouratidou, K., Chatzopoulos, D., Karamavrou, S. (2008). Validity study of the moral judgment test in physical education: development and preliminary validation. Perceptual Motor Skills, 106 (1), 51.

37. Núñez, J.L., Martín-Albo, J., Navarro, J.G., Sánchez, J.M., y GonzálezCutre, D. (2009). Intrinsic Motivation and Sportsmanship: Mediating Role of Interpersonal Relationships. Perceptual and Motor Skills, 108, 681-692.
38. Olivera, J. (2006). José María Cagigal y su contribución al humanismo deportivo. Revista Internacional de sociología, 64(44). Vol. LXIV, no 44 mayo-agosto, 207-235.

39. Ommundsen, Y., Roberts, G.C., Lemyre, P.N., y Treasure, D. (2003) Perceived motivational climate in male youth soccer: relations to socialmoral functioning, sportspersonship and team norm perceptions. Psychology of Sport and Exercise, 4(4), 397-413.

40. Proios, M. (2010). Development and validation of a questionnaire for the assessment of moral content judgment in sport. International Journal of Sport \& Exercise Psychology, 8(2), 189.

41. Pavlopoulou, E., Goniadou, S., Zachariadis, P., y Tsormpatoudis, H. (2003). The role of motivation to sportspersonship in physical education and sport. Hellenic Journal of Physical Education \& Sport, 48(2), 65-72.

42. Rodríguez, J. (coord) (2001). Estudio Social de la Zona Sur de Jerez. Grupo Universitario de Investigación Social. Jerez de la Frontera (Cádiz): Escuela Universitaria de Relaciones Laborales, Trabajo Social y Turismo.

43. Ryska, T. (2003). Sportsmanship in Young Athletes: The Role of Competitiveness, Motivational Orientation, and Perceived Purposes of Sport. Journal of Psychology, 137(3), 273.

44. Sáenz, A. (2010). Deportividad y violencia en el fútbol. (Tesis doctoral). Departamento de Psicología y Sociología, Universidad de Zaragoza, Zaragoza.

45. Serpa, S., Alves, P., y Barreiros, A. (2004). Versão portuguesa da Sport Motivational Scale (SMS) e da Sport Academic Scale (AMS): processos de tradução, adaptação e frabilidade. (Tese Doutorado).Faculdade de Motricidade Humana, Laboratório de Psicologia do Desporto. Universidade Técnica de Lisboa, Lisboa.

46. Sezen-Balçikanli, G. y Yildiran, I. (2011). Profesyonel futbolcularin sportmenlik yönelimleri ve empatik eğilim düzeyleri. SPORMETRE Be denEğitimiveSporBilimleriDergisi, 9(2) 49-56.

47. Shields, D.L., y Bredemeier, B.J. (1994). Character development and physical activity. Champaign, Illinois: Human Kinetics.

48. Shuge, Z. (2011). A study of sportsmanship and loyalty of athletic students and non-athletic students in hong kong baptist university. (Thesis). Baptisit University, Hong Kong.

49. Stornes, T. y Bru, E. (2002).Sportspersonship and perceptions of leadership.An investigation of adolescent handball players' perceptions of sportspersonship and relations with perceived leadership. European Journal of Sport Science, 6(2), 1-15.

50. Stornes, T., y Ommundsen, Y. (2004). Achievement goals, motivational climate and sportspersonship: a study of young handball players. Scandinavian Journal of Educational Research, 48(2), 205-221.

51. Vallerand, R.J., Brière, N.M., Blanchard, C., y Provencher, P. (1997) Development and validation of the multidimensional Sportspersonship orientations scale. Journal of Sport and Exercise Psychology, 19, 197-206.

52. Vissoci, J.R.N., Vieira, L.F., y Lopes, J.L. (2008). Motivação e atributos morais no esporte. Revista da Educação Física/UEM, 19(2), 173-182.

53. Widaman, K. F. (2007). Common factors versus components: Principals and principles, errors and misconceptions. En R. Cudeck y R. C. MacCallum (Eds.). Factor analysis at 100: Historical developments and future directions. Mahwah, NJ: LEA.

54. Zarotis, I.A., Psychountaki, M., y Stavrou, N. (2009, June). Study of Differences among Sex, Age and Type of Sport on Sportspersonship. Paper presented at the twelfth World Congress of Sport Psychology. Marrakesh- Morocco, Recuperado de http://karateeducatif.org/ SPORTS_PSYCHOLOGY/1\%20LONGS\%20ABSTRACT\%20 25\%20MAI/ORAL\%20y\%20POSTERS/Z/Zarotis,\%20I.A.,\%20Psychountaki,\%20M.,\%20Stavrou,\%20N.,\%20y\%20Zervas,\%20Y..pdf 